\title{
Detección de prescripciones potencialmente inapropiadas en pacientes ancianos: estudio descriptivo en dos farmacias comunitarias
}

\author{
Fernando Mud Castelló1, Sara Mud Castelló', María José Rodríguez Moncho', \\ María Dolores Ivorra Insa ${ }^{2}$, María Luisa Ferrándiz Manglano \\ 1. Farmacéuticos comunitarios en Ondara (Alicante). 2. Departamento Farmacología. Facultad de Farmacia. Universidad de Valencia.
}

\section{PALABRAS CLAVE}

farmacia comunitaria, Beers, STOPP, START, ancianos, polimedicados, prescripción potencialmente inadecuada, prescripción potencialmente omitida

\section{ABREVIATURAS}

STOPP: Screening Tool of Older Persons' potentially inappropriate Prescriptions. START: Screening Tool to Alert doctors to the Right Treatment. PPI: prescripciones potencialmente inadecuadas. PPO: prescripciomnes potencialmente omitidas. IBP: inhibidores de la bomba de protones. AINE: antiinflamatorios no esteroideos. CIE: clasificación internacional de enfermedades. ATC: clasificación anatómica, terapéutica y química de medicamentos.

\section{KEYWORDS}

community pharmacy, Beers, STOPP, START, elderly, polypharmacy, Potentially inappropriate prescription, potentially omitted prescriptions

\section{RESUMEN}

Introducción: Los ancianos son un grupo de pacientes heterogéneos a los que se les prescribe un número elevado de medicamentos. Esto conlleva a prescripciones potencialmente inapropiadas.

Objetivo: Analizar la farmacoterapia del paciente anciano desde la farmacia comunitaria para detectar prescripciones potencialmente inapropiadas (Beers 2012 y STOPP) y prescripciones potencialmente omitidas (START), determinando su prevalencia.

Metodología: Estudio descriptivo, observacional en el que se incluyeron pacientes mayores de 65 años de atención primaria que llevaban al menos un tratamiento crónico. Se verificó la idoneidad de la medicación según los criterios Beers 2012 y STOPP \& START.

Resultados: Se incluyeron 223 pacientes con una edad media de 75 años. En total se prescribieron 1.558 medicamentos, con una media de 7 medicamentos por paciente. El $67 \%$ de los pacientes eran polimedicados. Con Beers 2012 se detectaron 246 prescripciones inapropiadas y el criterio que más se repitió fue el de benzodiazepinas de acción corta, intermedia y larga (36\%). Con STOPP se detectaron 146 prescripciones inapropiadas. El criterio más frecuente fue IBP a dosis plenas durante más de 8 semanas (14\%). Con START se detectaron 103 prescripciones potencialmente omitidas, siendo antiagregantes plaquetarios en diabetes mellitus la más frecuente $(11,6 \%)$.

Conclusiones: Los criterios Beers 2012 y STOPP-START, suponen una herramienta de utilidad en la detección de los posibles problemas relacionados con los medicamentos en una farmacia comunitaria. En ningún caso suponen una prohibición en la utilización de dichos medicamentos, puesto que su prescripción dependerá de las características del paciente en concreto y del juicio clínico del médico prescriptor.

Detection of potentially inappropriate prescriptions for elderly patients: a descriptive study in two community pharmacies

\section{ABSTRACT}

Introduction: Elderly patients are a heterogeneous group who are prescribed a large number of medicaments. This leads to potentially inappropriate prescriptions.

Objective: To analyze the pharmacotherapy of elderly patients from community pharmacy in order to detect potentially inappropriate Prescriptions (STOPP and Beers 2012) and potentially omitted prescriptions (START), determining its prevalence.

Methodology: A descriptive, observational study in which patients over 65, who needed primary care and had at least one long-term treatment, were included. The appropriateness of the medicine was verified according to Beers 2012 and STOPP \& START criteria.

Results: 223 patients whose average age was 75 were included. 1558 medicines were prescribed in total, with an average of 7 medicaments per patient. $67 \%$ of patients were polypharmacy. With Beers 2012, 246 inappropriate prescriptions were detected and the most repeated criteria were that of Benzodiazepines: short, intermediate and long (36\%) effect. With STOPP 146 inappropriate prescriptions were detected. The most common criterion was full-doze IBP for more than 8 weeks (14\%). With START, 103 potentially omitted prescriptions were detected, being antiplatelet in diabetes mellitus the most frequent (11.6\%).

Conclusion: Beers and STOPP-START 2012 criteria represent a useful tool in the detection of potential drug-related problems in a community pharmacy. In no case they involve a ban on the use of these drugs, since its prescription depends on the characteristics of the individual patient and the clinical judgment of the prescribing physician.

Parte de este trabajo fue presentado en el V Congreso Nacional de Farmacéuticos Comunitarios (SEFAC) celebrado en Barcelona, en noviembre de 2012 y resultó finalista en la V Edición de los Premios Esteve. 


\section{Introducción}

Las personas mayores constituyen un grupo heterogéneo de pacientes donde se da un mayor grado de pluripatología y por tanto, de polimedicación, lo que genera un elevado coste para el Sistema Nacional de Salud (1) y puede además incrementar el número de reacciones adversas medicamentosas, interacciones, etc. Por ello, es necesario controlar estrechamente el uso de los medicamentos por este tipo de pacientes. Los principales problemas asociados con la prescripción en el anciano son los siguientes: infrautilización de medicación apropiada, sobreutilización, interacciones, falta de adherencia al tratamiento y el uso de medicación inapropiada (2-4).

Se considera que una prescripción es inapropiada cuando el riesgo de sufrir efectos adversos es superior al beneficio clínico, existiendo alternativas terapéuticas más seguras y/o eficaces. En el concepto se incluye el uso de fármacos con mayor frecuencia o duración de la indicada, con un elevado riesgo de interacciones, y la duplicidad de fármacos del mismo principio activo o clase (5).

Numerosos estudios documentan el uso de medicación inadecuada en ancianos (6). Se han desarrollado diversos criterios por parte de paneles de expertos en varios países para establecer la calidad de la prescripción y del uso de medicamentos en ancianos. Entre estos criterios los dos más utilizados, ambos para mayores de 65 años que llevan tratamiento crónico son los criterios de Beers (7-10) y los criterios STOPP (Screening Tool of Older Persons' potentially inappropriate Prescriptions) y START (Screening Tool to Alert doctors to the Right Treatment) (11-12).

En estos últimos años, diversos estudios han revisado el tratamiento farmacológico en ancianos europeos, utilizando para ello los criterios de Beers 2003 (11-18). Dichos estudios han detectado un número elevado de prescripciones potencialmente inapropiadas, con una prevalencia que va desde el 13\% (17) hasta el 29\% (15). Sin embargo, los resultados de estos trabajos son difíciles de comparar debido a las diferencias de población, metodología, y la proporción de criterios aplicados en cada estudio. Además, casi la mitad de los fármacos a los que hacen referencia los criterios Beers 2003 no están disponibles en todos los países de Europa y esto dificulta la transferencia de estos criterios entre países (19).

En muchos de estos estudios se aplican también los criterios STOPP $\mathrm{y}$ detectan un número mayor de prescripciones potencialmente inapropiadas, llegando incluso, en alguno de los estudios, a un 77-79\% de situaciones en las que no se debía de utilizar un determinado fármaco $(14,16)$. En población española se han realizado varios estudios en pacientes ancianos, aunque la mayoría de ellos revisan los tratamientos de ancianos institucionalizados $(14,20)$. Los resultados obtenidos son similares a los del resto de la población europea aplicando ambos tipos de criterios.

Cuando estos estudios aplican los criterios START para analizar las posibles omisiones en la prescripción, la prevalencia detectada está entre el 22-74\% (11,13,15-18).

En principio, estos estudios sugieren que con los criterios STOPP-START se identificarían más prescripciones potencialmente inapropiadas. Se siguen observando diferencias muy notables entre unos estudios y otros, lo que hace difícil extraer conclusiones que puedan llevar a la planificación de estrategias de mejora comunes. Sin embargo, sí pueden ser muy válidos para potenciar el análisis y la revisión de los tratamientos y poder im- plementar cambios que lleven a una mejor utilización de los medicamentos en la población anciana (21).

Existen pocos estudios que documenten la utilización de estos criterios en atención primaria en España, por lo que parece interesante aplicarlos, en farmacia comunitaria, con el objetivo de detectar prescripciones potencialmente inapropiadas (criterios Beers 2012 y STOPP) y prescripciones potencialmente omitidas (criterios START), determinando su prevalencia en pacientes mayores de 65 años.

\section{Material y métodos \\ Diseño}

Se trata de un estudio descriptivo, observacional, en el que se ha revisado la medicación de 223 pacientes de atención primaria que acuden a dos farmacias comunitarias en la provincia de Alicante (Comunidad Valenciana).

El criterio de inclusión era que el paciente tuviera 65 años o más, con, al menos, un tratamiento crónico y acudiese a la farmacia con la "hoja de crónicos". Se incorporaron al estudio todos los pacientes que durante siete días acudieron a la farmacia y cumplían los criterios de inclusión. La información de la hoja de crónicos que recibe el paciente se transcribió a una hoja de recogida de datos diseñada al efecto (figura 1).

DATOS DEL PACIENTE
\begin{tabular}{|l|l|l|}
\hline № & Sexo: & Edad:
\end{tabular}

Diagnósticos activos:

TRATAMIENTO FARMACOTERAPÉUTICO: Tratamiento crónico activo según la siguiente tabla:

\begin{tabular}{|c|c|c|c|c|c|c|c|c|}
\hline & Medicamento & Principio activo & Dosis & F. F. & Tiempo & Beers & STOPP & START \\
\hline \multicolumn{9}{|l|}{1} \\
\hline \multicolumn{9}{|l|}{2} \\
\hline \multicolumn{9}{|l|}{3} \\
\hline \multicolumn{9}{|l|}{4} \\
\hline \multicolumn{9}{|l|}{5} \\
\hline \multicolumn{9}{|l|}{6} \\
\hline \multicolumn{9}{|l|}{7} \\
\hline \multicolumn{9}{|l|}{8} \\
\hline \multicolumn{9}{|l|}{9} \\
\hline \multicolumn{9}{|l|}{10} \\
\hline \multicolumn{9}{|l|}{11} \\
\hline 12 & & & & & & & & \\
\hline
\end{tabular}

Figura 1 Hoja de recogida de datos 
Tabla 1 de la población en estudio
Caracteristicas

\begin{tabular}{|l|l|}
\hline Edad media & 75 años $(D E=9)$ \\
\hline Rango & $65-92$ años \\
\hline Sexo & $\begin{array}{l}\text { Hombres: } 91(41 \%) \\
\text { Mujeres: } 132(59 \%)\end{array}$ \\
\hline Medicación prescrita (total) & 1.558 \\
\hline Medicación media por paciente & $7(\mathrm{DE}=3)$ \\
\hline Rango & $1-18$ medicamentos \\
\hline Pacientes polimedicados (>6 medicamentos) & $149(67 \%)$ \\
\hline
\end{tabular}

Tabla 2 Número de pacientes y prevalencia de prescripciones potencialmente inapropiadas según Beers 2012 y STOPP, y de prescripciones potencialmente omitidas según START

\begin{tabular}{|l|c|c|c|}
\hline \multirow{2}{*}{ Prescripción inapropiada } & \multicolumn{3}{|c|}{ Pacientes $n(\%)$} \\
\cline { 2 - 4 } & Beers 2012 & STOPP & START \\
\hline Una & $66(29,0)$ & $74(33,2)$ & $38(17,0)$ \\
\hline Dos & $37(16,6)$ & $31(13,9)$ & $26(11,7)$ \\
\hline Tres o más & $30(13,5)$ & $7(3,0)$ & $5(2,3)$ \\
\hline Paciente con más criterios & 6 & 5 & 3 \\
\hline Pacientes con alguna & $133(60,0)$ & $112(50,2)$ & $69(30,9)$ \\
\hline
\end{tabular}

Tabla 3 Prescripciones potencialmente inapropiadas detectadas independientes del diagnóstico usando Beers 2012

Medicamentos

Anticolinérgicos (excluidos ATC)

Antihistamínicos de $1^{\text {a }}$ generación (mepiramina, hidroxizina)

Antiespasmódicos (trospio, solifenacina, otilonio bromuro, mebevirina)

Antitrombóticos

Dipiridamol de acción corta

Ticlopidina

Antibacterianos

Nitrofurantoina

Cardiovascular

Alfa-bloqueantes (doxazosina, terazosina)

Antiarritmicos (amiodarona, flecainida)

Digoxina $>0,125 \mathrm{mg} / \mathrm{dia}$

Espironolactona $>25 \mathrm{mg} / \mathrm{dia}$

Sistema nervioso central

Antidepresivos triciclicos (amitriptilina, nortriptilina)

Antipsicóticos: $1^{\text {a }}$ y $2^{\text {a }}$ generación (haloperidol, sulpirida)

Benzodiazepinas de acción corta (lorazepam, alprazolam, clotiazepam)

Benzodiazepinas de acción intermedia y larga (bromazepam, diazepam, medazepam, halazepam)

Hipnóticos no benzodiacepínicos (zolpidem)

Sistema endocrino

Estrógenos con o sin progestágenos (estriol, tibolona)

Insulina rápida

Dolor

AINE no selectivos (diclofenaco, ketoprofeno, ibuprofeno, metamizol)

16

Ketorolaco

Relajantes musculares (tetrazepam)

№ PPI: Número de prescripciones potencialmente inapropiadas.

\section{Datos analizados}

Se determinó la medicación potencialmente inadecuada aplicando los criterios Beers 2012 (10) y STOPP (11) y la medicación potencialmente omitida mediante los criterios START (12), de acuerdo con la propuesta de Mud et al (22) para la farmacia comunitaria, comparando los resultados obtenidos al aplicar cada uno de los criterios.

Los datos cualitativos se expresan como porcentajes y los cuantitativos como media \pm desviación estándar (DE).

\section{Resultados y discusión}

Características de los pacientes

En la tabla 1 se muestran las características de la población en estudio integrada por 223 pacientes.

\section{Prescripciones potencialmente} inapropiadas

En la tabla 2 se resumen el número de pacientes y la prevalencia de prescripciones potencialmente inadecuadas (PPI).

$\mathrm{Al}$ aplicar los criterios de Beers 2012 se detectaron 133 pacientes con al menos una PPI, con los criterios STOPP, la prevalencia de pacientes que presentaban al menos un criterio fue de 112 y según los criterios START 69 pacientes presentaban al menos una prescripción potencialmente omitida. En la tabla 2 se resume el número de pacientes y la prevalencia de prescripciones potencialmente inapropiadas según los criterios referidos.

Se describen a continuación las prescripciones potencialmente inapropiadas según los diferentes criterios utilizados.

Determinación de la prescripción potencialmente inadecuada utilizando los criterios de Beers 2012

En pacientes con al menos una prescripción potencialmente inapropiada se detectaron 28 criterios diferentes: 18 criterios eran independientes del diagnóstico o condición clínica (tabla 3), 7 criterios eran considerando el diagnóstico o condición clínica (tabla 4) y 3 criterios eran medicamentos que tienen que ser utilizados con precaución en ancianos (tabla 5). En las tablas se indica el número de veces que se repite cada criterio lo que supone un total de 246 prescripciones potencialmente inapropiadas. 
El criterio de Beers 2012 que más veces se repitió, teniendo en cuenta los criterios independientes del diagnóstico o condición clínica (tabla 3) era el benzodiazepinas de acción corta, intermedia y larga, que se repitió en 80 pacientes lo que representa un 36\%. En el presente estudio detectamos también un número elevado de PPI siguiendo los criterios del Anexo 3 (22) que recoge aquellos medicamentos que deben ser utilizados con precaución en el anciano (tabla 5).

Determinación de la prescripción potencialmente inadecuada utilizando los criterios STOPP

Como ya se ha comentado, se utilizaron también los criterios STOPP/START (5) para determinar las prescripciones inadecuadas (criterios STOPP). Los criterios STOPP comprenden 65 criterios divididos en 10 grupos: 7 sistemas fisiológi$\cos (A-G), 2$ grupos de medicamentos $(\mathrm{H}, \mathrm{I})$ y el criterio de duplicidad (J).

En la tabla 6 se muestra el número de prescripciones potencialmente inadecuadas detectadas en la población en estudio al aplicar los criterios STOPP. Se detectaron un total de 146 prescripciones potencialmente inadecuadas agrupadas en 28 criterios STOPP diferentes: 9 correspondian a prescripciones potencialmente inadecuadas del sistema cardiovascular (A), 6 del sistema nervioso central y psicofármacos (B), 1 del sistema gastrointestinal (C), 7 del sistema musculoesquelético (E), 1 del sistema urogenital (F), 2 del sistema endocrino (G), 1 de analgésicos (I) y 1 a medicamento duplicado (J).

El criterio STOPP que más veces se repitió fue el C4, IBP para la enfermedad ulcerosa péptica a dosis terapéuticas plenas durante más de 8 semanas; se detectó en 31 pacientes, lo que representa un 14\% del total de la población estudiada. Este resultado coincide con lo descrito en otros estudios en diferentes poblaciones ancianas $(14,15,17)$. El criterio B7 uso prolongado de benzodiazepinas de vida media larga o benzodiazepinas con metabolitos de larga acción, junto con el criterio B13 uso prolongado (más de 1 semana) de antihistaminicos de primera generación, ocupan el segundo

Tabla 4 Prescripciones potencialmente inapropiadas detectadas considerando el diagnóstico usando Beers 2012

\begin{tabular}{|c|l|c|}
\hline Enfermedad & Medicamentos & Cantidad \\
\hline Sistema nervioso central & & 3 \\
\hline Fallo cardiaco & Verapamilo, diltiazem & 1 \\
\hline Delirio & Benzodiazepinas (lorazepam) & \\
\hline Demencia y daño cognitivo & $\begin{array}{l}\text { Benzodiazepinas (bromazepam, } \\
\text { lorazepam) }\end{array}$ & 2 \\
\hline Insomnio & Antipsicóticos (risperidona) & 1 \\
\hline Gastrointestinal & Cafeína & 1 \\
\hline Estreñimiento & Antipsicóticos: haloperidol & \\
\hline Antecedentes de úlcera gástrica o duodenal & $\begin{array}{l}\text { AlNE no selectivos (ibuprofeno, } \\
\text { diclofenaco, ketoprofeno) }\end{array}$ & 6 \\
\hline Hiperplasia benigna de próstata & Tiotropio & 1 \\
\hline TOTAL & & 15 \\
\hline
\end{tabular}

Tabla 5 Prescripciones potencialmente inapropiadas (PPI) detectadas considerando medicamentos que tienen que ser utilizados con precaución en el anciano usando Beers 2012

$\begin{array}{lll}\text { Medicamento } & \text { No PP }\end{array}$

Aspirina para prevención primaria de eventos cardiacos

Antipsicóticos (haloperidol, risperidona, sulpirida, perfenazina)

Inhibidores recaptación serotonina y noradrenalina (maprotilina)

Inhibidores selectivos de la recaptación serotonina

(sertralina, fluoxetina, paroxetina, escitalopram, citalopram)

Antidepresivos tricíclicos (amitriptilina, nortriptilina)

Mirtazapina

Vasodilatadores (nitroglicerina) 2

TOTAL

y tercer lugar detectándose en 27 pacientes (12,1\%). Ambos grupos de medicamentos tienen riesgo de producir sedación prolongada y riesgo de caídas por lo que se suman sus efectos adversos, problema que también se recogen en los resultados de otros autores $(11,13,16)$.

\section{Prescripciones}

potencialmente omitidas

Se aplicaron los criterios START que comprenden 22 criterios repartidos en 6 sistemas fisiológicos (Anexo 5).

Se detectó un total de 103 prescripciones potencialmente omitidas agrupadas en 19 criterios START diferentes: 8 correspondían a tratamientos indicados y apropiados del sistema cardiovascular (A), 2 del sistema respiratorio (B), 2 del sistema nervioso central (C), 3 el sistema musculoesquelético (E) y 4 del sistema endocrino (F) (tabla 7)
El criterio START que más veces se repitió fue el F3, antiagregantes plaquetarios en la diabetes mellitus si coexisten uno o más factores mayores de riesgo cardiovascular (hipertensión, hipercolesterolemia, consumo de tabaco). Se produjo en 26 pacientes lo que representa un 11,6\%. En segundo lugar se encuentra la omisión de la prescripción de estatinas (con antecedentes bien documentados de enfermedad arteriosclerótica) (criterio F4) que se repitió en 13 pacientes, lo que representa un 5,8\% del total de pacientes. Esto coincide con otros estudios donde las omisiones más frecuentes también son criterios del sistema endocrino (17). Otro criterio que también aparece con relativa frecuencia en nuestro estudio es la omisión de suplementos de calcio y vitamina D (en pacientes con osteoporosis conocida) (criterio E3). Esto coincide con otros estudios en pacientes ancianos tanto europeos (13) como españoles (14) 
Tabla 6 Prescripciones potencialmente inapropiadas detectadas usando STOPP

Sistema cardiovascular

A1. Digoxina a dosis superiores $123 \mathrm{mg} /$ dia a largo plazo en presencia insuficiencia renal

A2. Diuréticos de asa para los edemas maleolares aislados, sin signos clínicos de IC

A3. Diuréticos de asa (torasemida) como monoterapia de primera línea en la hipertensión (existen alternativas más seguras y eficaces)

A4. Diuréticos tiazídicos (hidroclorotiazida) con antecedentes de gota (pueden exacerbar la gota)

A7. Uso de diltiazem o verapamilo en la insuficiencia cardíaca grado III o IV de la NHYA

A8. Antagonistas del calcio en el estreñimiento crónico (pueden agravar el estreñimiento) (amlodipino, manidipino)

A12. AAS a dosis superiores a $150 \mathrm{mg}$ día (aumento del riesgo de sangrado, sin evidencia de una mayor eficacia)

A13. AAS sin antecedentes de cardiopatía isquémica, enfermedad cerebrovascular, enfermedad arterial periférica o un antecedente oclusivo arterial (no indicada)

A14. AAS para tratar un mareo no claramente atribuible a enfermedad cerebrovascular

Sistema nervioso central y psicofármacos

B3. ATC (amitriptilina) con trastornos de la conducción cardiaca (efectos proarrítmicos)

B5. ATC (amitriptilina, nortriptilina) con opiáceo o un antagonista del calcio (riesgo de estreñimiento grave)

B7. Uso prolongado (i.e. más de 1 mes) de benzodiazepinas de vida media larga (bromazepam, medazepam, halazepam) o benzodiazepinas con metabolitos de larga acción (como diazepam) (riesgo de sedación prolongada, confusión, trastornos del equilibrio, caídas)

B8. Uso prolongado (i.e. más de 1 mes) de neurolépticos (haloperidol, risperidona, sulpirida, perfenazina) como hipnóticos a largo plazo (riesgo de confusión, hipotensión, efectos extrapiramidales, caídas)

B12. ISRS (fluoxetina, paroxetina, escitalopram) con antecedentes de hiponatremia clínicamente significativa (hiponatremia inferior a $130 \mathrm{mmol} / \mathrm{L}$ no iatrogénica en los dos meses anteriores)

B13. Uso prolongado (más de 1 semana) de antihistamínicos de primera generación, (mepiramina, hidroxizina) (riesgo de sedación y efectos secundarios anticolinérgicos)

\section{Sistema gastrointestinal}

C4. IBP (pantoprazol, lansoprazol, omeprazol, esomeprazol) para la enfermedad ulcerosa péptica a dosis terapéuticas plenas durante más de 8 semanas (está indicada la suspensión o descenso de dosis más precoz para el tratamiento de mantenimiento/ profiláctico de la enfermedad ulcerosa péptica, la esofagitis o la enfermedad por reflujo gastroesofágico)

Sistema musculoesquelético

E1. AINE con antecedentes de enfermedad ulcerosa péptica o hemorragia digestiva, salvo con uso simultaneo de antagonistas $\mathrm{H} 2$, IBP o misoprostol

E2. AINE (ibuprofeno) con hipertensión moderada-grave (moderada: 160/100 mmHg-179/109 mmHg; grave: igual o superior a 180/110 mmHg) (riesgo de empeoramiento de la hipertensión)

E3. AINE con insuficiencia cardiaca

E4. Uso prolongado de AINE (más de 3 meses) en dolor articular en la artrosis

E6. AINE con insuficiencia renal crónica

E7. Corticosteroides (prednisona) a largo plazo (más de 3 meses) como monoterapia para la artritis reumatoide o la artrosis (riesgo de efectos secundarios sistémicos mayores de los corticoides)

E8. AINE o colchicina para tratamiento crónico de la gota cuando no existe contraindicación al alopurinol

\section{Sistema urogenital}

F4. Fármacos antimuscarínicos (solifenacina) vesicales con prostatismo crónico (riesgo de retención urinaria)

\section{Sistema endocrino}

G1. Glibenclamida con diabetes mellitus tipo2 (riesgo de hipoglucemia prolongada)

G2. Bloqueadores beta (nebivolol) en la diabetes mellitus con frecuentes episodios de hipoglucemia, i.e. 1 o más episodios al mes (riesgo de enmascaramiento de los síntomas de hipoglucemia)

\section{Analgésicos}

12. Opiáceos regulares durante más de dos semanas en aquellos con estreñimiento crónico sin uso de laxantes

J. Clase de medicamento duplicada 
Sistema cardiovascular

A1. Warfarina en presencia de una fibrilación auricular crónica

A2. AAS en presencia de una fibrilación auricular crónica, cuando la warfarina esté contraindicada pero no lo esté el AAS

A3. AAS o clopidogrel con antecedentes bien documentados de enfermedad arteriosclerótica coronaria, cerebral o arterial periférica en pacientes en ritmo sinusal

A4. Tratamiento antihipertensivo cuando la presión arterial sistólica sea normalmente superior a $160 \mathrm{mmHg}$

A5. Estatinas con antecedentes bien documentados de enfermedad arteriosclerótica coronaria, cerebral o arterial periférica, cuando la situación funcional sea de independencia para las actividades básicas de la vida diaria y la esperanza de vida superior a 5 años

A6. IECA en la insuficiencia cardiaca crónica

A7. IECA tras un infarto agudo de miocardio

A8. Bloqueadores beta en angina crónica estable

Sistema respiratorio

B2. Corticosteroide inhalado pautado en el asma o la EPOC moderada a grave, cuando la FEV1 es inferior al 50\%

B3. Oxigenoterapia domiciliaria continua en la insuficiencia respiratoria tipo 1 ( $\mathrm{pO} 2<8,0 \mathrm{kPa}$ [60 mmHg], pC02 <6,5 kPa [49 $\mathrm{mmHg}$ ) o tipo 2 (p02 <8,0 kPa [60 mmHg], pCO2 46,5 kPa [49 mmHg]) bien documentada

Sistema nervioso central

C1. Levodopa en la enfermedad del Parkinson idiopática con deterioro funcional evidente

C2. Antidepresivos en presencia de sintomas depresivos moderados a graves durante al menos tres meses

Sistema musculoesquelético

E1. Fármacos antirreumáticos modificadores de la enfermedad en la artritis reumatoide moderada a grave activa de más de 12 semanas de duración

E2. Bifosfonatos en pacientes que reciben corticosteroides orales a dosis de mantenimiento

\section{Limitaciones del estudio}

La muestra corresponde a todos los pacientes mayores de 65 años atendidos en las dos farmacias comunitarias participantes. No se ha realizado el cálculo de tamaño muestral para conseguir representatividad, por lo que los resultados son válidos únicamente para la población atendida en ambas farmacias.

En los criterios STOPP, el grupo $\mathrm{H}$, Fármacos que afectan negativamente a los propensos a caerse (1 o más caídas en los últimos tres meses) no se incluyeron en el estudio, ya que los pacientes eran ambulatorios y el médico no disponía en la historia clínica de los datos de caídas. Lo mismo ocurrió con el criterio History of falls or fractures de Beers 2012.

Uno de los aspectos que nos hubiera gustado analizar era la relación entre el medicamento prescrito y la indicación por la que se prescribe para comprobar que todos los medicamentos tuvieran una indicación clara y descartar que la indicación no sea por una reacción adversa producida por otro fármaco. Finalmente no se pudo llevar a cabo porque los médicos ambulatorios no incluyen en la hoja de crónicos todos los diagnósticos que realmente tienen sus pacientes. La explicación es muy fácil, no disponen de tiempo en la consulta, y engloban varios diagnósticos en uno solo, lo que les permite ahorrar tiempo y así dedicarle más al paciente. Además hay enfermedades diagnosticadas en el hospital que el médico ambulatorio sabe que las tiene el paciente, pero no están en la base de datos del Abucasis (programa informático usado en los ambulatorios del Servicio Público de Salud de la Comunidad Valenciana).

Ninguno de los criterios usados en el estudio presenta los grupos terapéuticos o principios activos según la clasificación ATC. En algunos casos sólo pone el grupo de fármacos y en otros casos detalla ejemplos de moléculas de esos grupos; esto puede hacer dudar, al aplicar estos criterios, si incluir solo las citadas en el ejemplo o todas las que forman el grupo.

En los criterios de Beers, 2012, en el apartado de criterios según diagnóstico hay ciertas enfermedades que son muy generales, por ejemplo: daño cognitivo. La interpretación de qué enfermedades agrupa este criterio puede variar según la persona que lo aplica. Sería muy beneficioso clasificar las enfermedades de los criterios según la clasificación internacional de las enfermedades (CIE), ya que así evitariamos la libre interpretación y además, facilitaría mucho la aplicación de los criterios porque en las hojas de tratamientos crónicos que llevan los pacientes, las enfermedades se presentan por código CIE. 


\section{Conclusiones}

Se han detectado un número elevado de PPI: 246 con los criterios Beers 2012 y 146 con STOPP. También se han detectado 103 PPO según los criterios START.

Beers 2012 detecta más prescripciones potencialmente inapropiadas, siendo diferentes para cada tipo de criterio: la utilización de BDZ con Beers 2012 y los IBP a dosis plenas durante 8 semanas con los STOPP. Con respecto a las PPO, la más frecuente es "antiagregantes plaquetarios en diabetes mellitus".

La aplicación de todos ellos supone una herramienta de utilidad en la detección de los posibles problemas relacionados con los medicamentos en pacientes ancianos en una farmacia comunitaria.

Es necesaria la implementación de estrategias para identificar las prescripciones inapropiadas y para conseguir un uso adecuado de los fármacos en pacientes de edad avanzada.

En ningún caso cualquiera de los criterios que apliquemos suponen una prohibición en la utilización de dichos medicamentos, puesto que su prescripción siempre dependerá de la situación clínica y características del paciente en concreto, así como del juicio clínico del médico prescriptor.

\section{Referencias bibliográficas}

1. Instituto de Información Sanitaria [Internet]. Mayo 2005. [Acceso 20/7/13]. Disponible en: http://www. msps.es/estadEstudios/estadisticas/ docs/Consumo_farmaceutico_por_ tramos_edad_y_sexoII_Final.pdf

2. Información Farmacoterapéutica de la Comarca: Medicación en el anciano. Infac. 2009;17(6):1-6.

3. Rochon PA. Drug prescribing for older adults [Internet]. Actualizada Mayo 2012. [Acceso: 6/6/2012]. Disponible en: http://www.uptodate.com/ contents/drug-prescribing-for-older-adults? source = search re sultctsearch $=d$ rug + prescription+for+older+adultsCtselectedTitle =1 150

4. Rochon PA, Geoffrey M. Prescribing Optimal Drug Therapy for Older People. Arch Intern Med. 2010;170(1):103-106. 4. Rochon PA, Geoffrey M. Prescribing Optimal Drug Therapy for Older People. Arch Intern Med. 2010;170(1):103-106. doi:10.1001/archinternmed.2009.473

5. Delgado-Silveira E, Muñoz-García M, Montero-Errasquin B, Sánchez-Castellano C, Gallagher P, Cruz-Jentoft AJ. Prescripción inapropiada de medicamentos en los pacientes mayores: los criterios STOPP/START. Rev Esp Geriatr Gerontol. 2009;44(5):273279. doi:10.1016/j.regg.2009.03.017

6. Laroche ML, Charmes JP, Bouthier F, Merle L. Inappropriate Medications in the Elderly. Clin Pharmacol Ther. 2009;85:94-97. doi:10.1038/ clpt.2008.214

7. Beers MH, Ouslander JG, Rollingher J, Reuben DB, Brooks J, Beck J. Explicit criteria for determining inappropriate medication use in nursing home. Arch lntern Med. 1991;151:1825-1832. doi:10.1001/ archinte.1991.00400090107019

8. Beers MH. Explicit Criteria for Determining Potentially Inappropriate Medication Use by the Elderly. Arch Intern Med. 1997;157(14):1531-1536. doi:10.1001/ archinte.1997.00440350031003

9. Fick DM, James W, Cooper R, William E, Wade JL, Waller J, et al. Updating the Beers Criteria for Potentially Inappropriate Medication Use in Older Adults. Arch Intern Med. 2003;163:2716-2724. doi:10.1001/ archinte.163.22.2716

10. American Geriatrics Society. Updated Beers Criteria for Potentially Inappropriate Medication Use in Older Adults. J Am Geriatr Soc. 2012;60(4):616-631. doi:10.1111/j.1532-5415.2012. 03923.x

11. Gallagher P, O'Mahony D. STOPP (Screening Tool of Older Persons' potentially inappropriate Prescriptions): application to acutely ill elderly patients and comparison with Beers' criteria. Age Ageing. 2008;37: 673-679. doi:10.1093/ageing/afn 197

12. Barry PJ, Gallagher P, Ryan C, O'Mahony D. START (Screeníng Tool to Alert doctors to the Right Treatment: an evidence-based screening tool to detect prescribing omissions in elderly patients. Age Ageing. 2007;36:632-638. doi:10.1093/ageing/afm 118

13. Gallagher P, Lang OP, Cherubini A, Topinková E, Cruz-Jentoft A, Montero Errasquín B, et al. Prevalence of potentially inappropriate prescribing in an acutely ill population of older patients admitted to six European hospitals. Eur J Clin Pharmacol. 2011; 67:1175-1188. doi:10.1007/s00228011-1061-0

14. Garcia-Gollarte F, Baleriola-Júlvez J, Ferrero Lopez I, Cruz-Jenfot A. Inappropriate Drug Prescription at Nurs- ing Home Admission. J Am Med Dir Assoc. 2012;13(1):83.e9-15.

15. Hamilton H, Gallagher P, Ryan C, Byrne S, O’Mahony D. Potentially Inappropriate Medications Defined by STOPP Criteria and the Risk of Adverse Drug Events in Older Hospitalized Patients. Arch Intern Med. 2011;171(11):1013-1019. doi:10.1001/ archinternmed.2011.215

16. Lang PO, Hasso $\mathrm{Y}$, Dramé M, Vogt-Ferrier N, Prudent M, Gold G, et al. Potentially inappropriate prescribing including under-use amongst older patients with cognitive or psychiatric co-morbidities. Age Ageing. 2010;39:373-381. doi:10.1093/ageing/afq031

17. Ryan C, O’Mahony D, Kennedy J, Weedle P, Stephen B. Potentially inappropriate prescribing in an Irish elderly population in primary care. $\mathrm{Br} \mathrm{J}$ Clin Pharmacol. 2009;68(6):936-947. doi:10.1111/j.1365-2125.2009.03531.x

18. Ryan C, O'Mahony D, Kennedy J, Weedle P, Barry P, Gallagher P, et al. Appropriate prescribing in the elderly: an investigation of two screening tools, Beers criteria considering diagnosis and independent of diagnosis and improved prescribing in the elderly tool to identify inappropriate use of medicines in the elderly in primary care in Ireland. J Clin Pharm Ther. 2009;34:369-376. doi:10.1111/ j.1365-2710.2008.01007.x

19. Spinewine A, Schmader KE, Barber N, Hughes C, Lapane KL, Swine C, Hanlon JT. Appropriate prescribing in elderly people: how well can it be measured and optimised? Lancet 2007;370:173-184. doi:10.1016/ S0140-6736(07)61091-5

20. Úbeda $A$, Ferrándiz ML, Maicas N, Gómez C, Bonet M, Peris JE. Potentially inappropriate prescribing in institutionalized older patients in Spain: the STOPP-START criteria compared with the Beers criteria. Pharmacy Practice (Internet). 2012 Apr-Jun;10(2):83-91. doi:10.4321/ S1886-36552012000200004

21. Tangiisuran B, Wright J, Van der Cammen T, Rajkumar Ch. Adverse drug reactions in elderly: Challenges in identification and improving preventative strategies. Age Ageing. 2009;38:358-359. doi:10.1093/ageing/afp050

22. Mud Castelló F, Mud Castelló S, Rodríguez Moncho MJ, Insa MD, Ferrándiz Manglano ML. Herramientas para evaluar la adecuación de la prescripción en ancianos. Farmacéuticos Comunitarios. 2013;5(4):147-51. 